\title{
Estimating Ground Inclination Using Strain Sensors with Fourier Series Representation
}

\author{
Wolfgang Svensson and Ulf Holmberg \\ Intelligent System Laboratory, IDE Department, Halmstad University, Box 823, 30118 Halmstad, Sweden \\ Correspondence should be addressed to Ulf Holmberg, ulf.holmberg@hh.se
}

Received 1 November 2009; Accepted 25 January 2010

Academic Editor: Noriyasu Homma

Copyright ( $) 2010$ W. Svensson and U. Holmberg. This is an open access article distributed under the Creative Commons Attribution License, which permits unrestricted use, distribution, and reproduction in any medium, provided the original work is properly cited.

\begin{abstract}
An embedded measurement system for foot orthosis during gait is proposed. Strain gauge sensors were mounted on a foot orthosis to give information about strain in the sagittal plane. The ankle angle of the orthosis was fixed and strain characteristics were therefore changed when walking on slopes. With a Fourier series representation of the strain during a gait cycle, ground angle at different walking speeds and inclinations could be estimated with similar accuracy as previous studies using kinematically based estimators. Furthermore, if the angle of the mechanical foot ankle was changed, the sensing technique still could estimate ground angle without need for recalibration as opposed to kinematical sensors. This indicates that embedded strain sensors can be used for online control of future orthoses with inclination adaptation. Also, there would be no need to recalibrate the sensing system when changing shoes with different heel heights.
\end{abstract}

\section{Introduction}

Ankle and foot muscles disorders affect the human gait and are commonly treated with orthoses to partially compensate functional loss. With an ankle-foot orthosis, AFO, typical assisting functions are provide ankle stability during stance, stimulate push-off effect during late stance, keep the toes off ground during swing, assist poor functional muscles, and decrease pain by limiting motion. Orthoses have been passive and purely mechanical. But decreasing size and cost of electronics have made it possible for active solutions of assistance, for example, [1-3]. But existing systems are still limited in their capability of adapting to new inclining circumstances, that is, hills or heel height variation.

Studies have shown that when able-bodied walked uphill, gait was adjusted in the ankle foot system [4]. Downhill walking is considered to be different in the sense that joints have to absorb more energy caused by the combined forward and downward movement (see, e.g., [5-7]). This can be seen by a shorter stride length [5] and is compensated mostly by the knees and somewhat by the hip [4].

It is stated in [8] that people with reduced range of motion in one joint compensate by using their other joints.
But with elderly, orthotic, and prosthesis users, these changes in flexion may not be possible. Many of the assisting devices have fixed ankle position and attempting to move the body's center of mass forward may cause a sense of instability when walking in inclinations. Our hypothesis is that this restricted adaptation possibility causes an extra torque on the AFO. This would therefore be measurable and useful for estimating the ground angle. In the future this could be used in ankle control for adapting to the ground inclination.

Portable gait measuring techniques are interesting both for gait analysis and active control. Automatic classification of gait phases has previously been done using various wearable sensors, for example, force resistive sensors (FSR) $[9,10]$, gyros [11], combinations of FSRs and gyros [12], accelerometers [13-15], or goniometers [16]. It has also been shown that upper-body mounted accelerometers together with artificial neural networks can be used for slope and speed estimation. Aminian et al. [17] used summary statistics (median, variance, etc.) for inclination estimation and Wang et al. [18] used wavelets to separate slope from level walking. Sabatini et al. [15] mounted accelerometers on the foot and used them as gravity sensors during stance. 


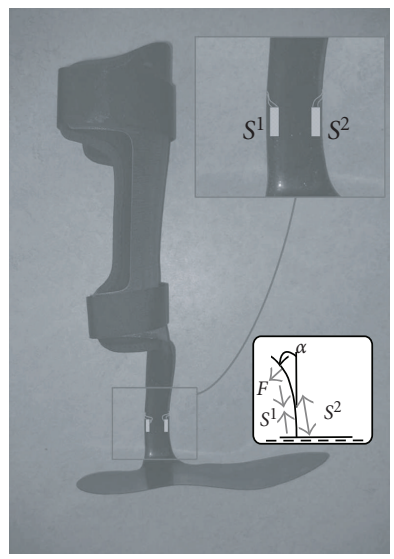

(a)

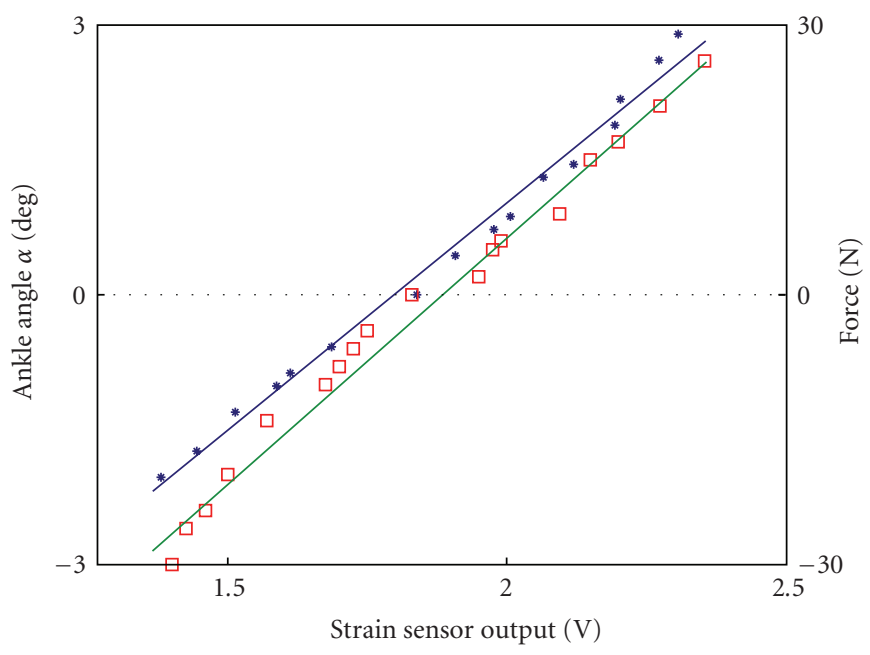

(b)

Figure 1: (a) Strain measurement setup. One strain gauge sensor $S^{1}$ is attached partly on the inside at the back and one $S^{2}$ partly on the inside at the front. Sensors $S^{3}$ and $S^{4}$ are attached at the corresponding positions but on the outside. (b) Measured ankle angle $\alpha$ (stars) and force $F$ (squares) relation to AFO strain sensor output. Estimated linear relations: solid $(\alpha)$ and dashed (F).

Torque is typically estimated from measuring ground reaction forces and ankle angle. Instead of using previously mentioned sensors, the use of strain gauge sensors as an indirect measurement is investigated. Another feature of strain sensors is the small size that allows embedded constructions (see Figure 1). These sensors can be valuable for active control as well as for prosthetic and orthotic design.

Previous studies showed that gait cycle strain peak and mean value changed in inclination [19]. But similar changes in strain can also be caused by a changed gait speed. In this work we propose to take advantage of the fact that gait cycles are periodic. A Fourier series representation can then be used to code the information into fewer variables. This is then used for estimation of the ground inclination. It extends the work in [19] and shows that a fixed estimation model can be used even though the speed changes. In addition and more importantce, the estimation works also when the ankle angle is changed. This indicates that online ankle angle control would be possible using the strain signal and that no recalibration would be necessary when changing shoes to other heel heights.

\section{Strain Measurement}

2.1. Strain Sensor Setup. A solid light-weight carbon anklefoot orthosis was used. Four strain gauge sensors were glued $60 \mathrm{~mm}$ above the orthotic sole as shown in Figure 1(a). The sensor signals were combined into one via a (full Wheatstone) bridge. The combined amplified sensor signal was sampled with an off-the-shelf PIC processor with a 10bit $\mathrm{AD}$-converter at $50 \mathrm{~Hz}$ sampling frequency. The signal was logged into a PC with Sysquake software using Bluetooth modules. The strain sensor signals are combined into one strain signal describing the angle behavior in the sagittal (vertical-longitudinal) plane during gait.

A torque applied on an AFO can be modeled as a force $F$, acting on a beam with one fix end as shown in Figure 1(a), where the beam bending angle $\alpha$ is proportional to the force for small angles. Instead of measuring the angle $\alpha$ and the force $F$ or trying to reconstruct the corresponding torque, the attached strain sensors are used to produce information about the orthosis bending during gait.

Experiments were made to verify the strain signal being proportional to both applied force $F$ and angle $\alpha$ in Figure 1(b).

2.2. Strain Signal Behavior. From Figure 1(b) it is seen that no strain (when the orthosis is unloaded) corresponds to a sensor output around 1.8 Volts. The sensor signal increased at plantar flexion and decreased at dorsiflexion; see Figure 2. At heel strike the strain increased to a peak from being unloaded as the foot was put down in front of the body. The foot was then lowered and the trunk moved forward decreasing the strain. The body continued forward and the leg tried to rotate with an ankle dorsiflexion. An oscillation in the sensor signal was sometimes seen around the time of heel lift. In the end of the stance phase, an abrupt decrease of the strain signal occurred. This was the largest load on the orthosis caused by the push-off. After toeoff, the inherent orthotic elasticity brought the orthosis to unload. Although the sensor setup was designed to mainly measure in the sagittal (vertical-longitudinal) plane it was also somewhat influenced by the natural motion in the coronal (vertical-transversal) plane during weight acceptance. 


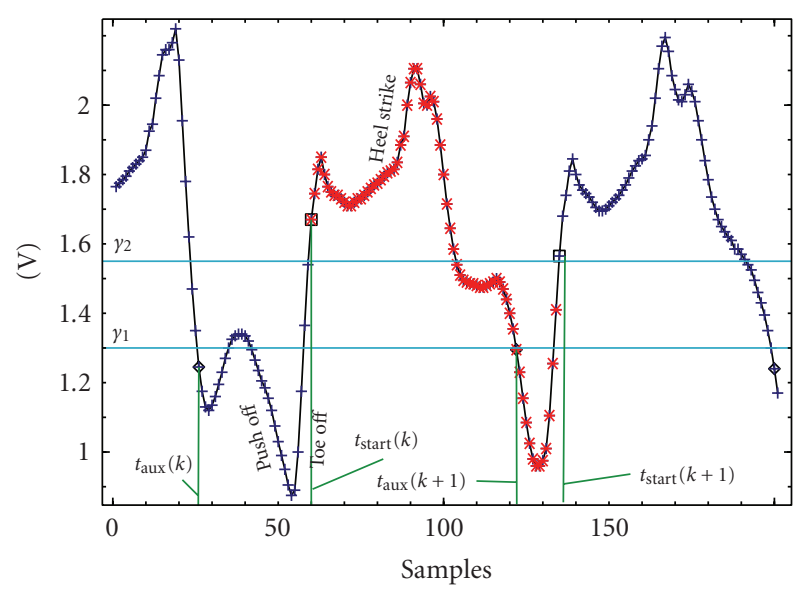

FIGURE 2: AFO strain signal output and extraction of strain signal for one gait cycle $($ red $(*))$. Thresholds $\gamma_{1}, \gamma_{2}$ for finding the auxiliary times $t_{\text {aux }}$ (first $t$ when $S(t)<\gamma_{1}$ ) and $t_{\text {start }}$ (first $t$ when $\left.S(t)>\gamma_{2}\right)$ are indicated.

\section{Methods}

3.1. Strain Signal Extraction. In order to extract one gait cycle from the strain sensor output, an appropriate starting point is selected to be particularly easy to detect. This is chosen to be after toe-off since here the strain sensor signal is making an abrupt monotonous increase. However, there are sometimes oscillations around heel lift with the sensor signal increasing which should not be misinterpreted as toe lift (see Figure 2). By introducing two thresholds, the time instant just after toe-off can be found. The procedure is as follows.

Denote the strain sensor output $S(t)$ with sample index $t$ (integer). Let $t_{\text {start }}(0)$ be any initial sampling instant. Find the start of gait cycle $k$, defined at $t_{\text {start }}(k)$ by using auxiliary sampling instant $t_{\text {aux }}(k)$, according to

$$
\begin{gathered}
t_{\text {aux }}(k)=\min _{t>t_{\text {start }}(k-1)}\left\{t \mid S(t)<\gamma_{1}\right\}, \\
t_{\text {start }}(k)=\min _{t>t_{\text {aux }}(k)}\left\{t \mid S(t)<\gamma_{1}\right\} .
\end{gathered}
$$

The strain signal (vector) at gait cycle $k$ is now defined as

$$
\mathbf{s}_{k}=\left(\begin{array}{c}
s_{1} \\
s_{2} \\
\vdots \\
s_{N}
\end{array}\right)=\left(\begin{array}{c}
S\left(t_{\text {start }}(k)\right) \\
S\left(t_{\text {start }}(k)+1\right) \\
\vdots \\
S\left(t_{\text {start }}(k+1)-1\right)
\end{array}\right) .
$$

The index $k$ will be omitted for simplicity except when the gait number is specific for the expression.

3.2. Fourier Series Representation. Since the dimension $(N)$ of the strain vector is changing with the walking speed, it is inconvenient to use the strain vector $\mathbf{s}$ directly in an estimation scheme. However, due to the periodicity of the strain with $S(t+N)=S(t)$, it would make sense to use Fourier series representation. Then the gait information in the strain signal is coded into fewer variables efficiently and with a vector representation with a fixed prechosen dimension.

Using $n$ harmonics, the Fourier coefficients can be written in matrix form as

$$
x=\left(\begin{array}{l}
a_{0} \\
a \\
b
\end{array}\right)=\left(\begin{array}{c}
\frac{1}{N} \sum_{i=1}^{N} s_{i} \\
\frac{2}{N} e^{T} \mathbf{s} \\
\frac{2}{N} \delta^{T} \mathbf{s}
\end{array}\right),
$$

where the matrixes $\mathcal{C} \in \mathfrak{R}^{N \times n}$ and $\delta \in \mathfrak{R}^{N \times n}$ have the elements $\mathcal{C}_{i j}=\cos (i j \Omega), \wp_{i j}=\sin (i j \Omega)$, and $\Omega=2 \pi / N$. Letting $\mathbf{1}=[1 \cdots 1]^{T} \in \mathfrak{R}^{N \times 1}$, the basis functions can be put in a matrix where each column corresponds to a basis function

$$
E=\left(\begin{array}{lll}
1 & C & \delta
\end{array}\right) \in \mathfrak{R}^{N \times(2 n+1)} .
$$

The strain can then be approximated as

$$
\widehat{\mathbf{s}}=E x \in \mathfrak{R}^{N \times 1}
$$

which is the orthogonal projection to the space spanned by the $2 n+1$ basis functions, that is,

$$
x=\arg \min _{x}\|\mathbf{s}-\hat{\mathbf{s}}\|^{2} \in \mathfrak{R}^{2 n+1} .
$$

Notice that the dimension of $x$ is fixed and chosen $(=2 n+1)$ and usually much smaller than the dimension of $\mathbf{s}(=N)$, which also varies from step to step.

3.3. Estimation of Inclination. Assume that the ground angle $\phi$ can be modeled linearly by the parameter vector $\theta$ from the Fourier coefficients $x$ as

$$
\phi_{k}=x_{k}^{T} \theta+\epsilon_{k}
$$

where $\epsilon_{k}$ is stochastic white noise and

$$
\theta=\left(\begin{array}{lll}
\theta_{1} & \cdots & \theta_{2 n+1}
\end{array}\right)^{T} .
$$

The model parameters $\theta$ are found using $M$ steps data

$$
\begin{aligned}
\phi & =\left(\begin{array}{lll}
\phi_{1} & \cdots & \phi_{M}
\end{array}\right)^{T}, \\
X & =\left(\begin{array}{lll}
x_{1} & \cdots & x_{M}
\end{array}\right)^{T} \longrightarrow \phi=X \theta+\epsilon, \\
\epsilon & =\left(\begin{array}{lll}
\epsilon_{1} & \cdots & \epsilon_{M}
\end{array}\right)^{T}
\end{aligned}
$$

by the least squares method as

$$
\theta=\arg \min _{\theta}\left(\epsilon^{T} \epsilon\right)=\left(X^{T} X\right)^{-1} X^{T} \phi .
$$

Inclination can then be estimated from strain according to

$$
\hat{\phi}_{k}=x_{k}^{T} \theta
$$




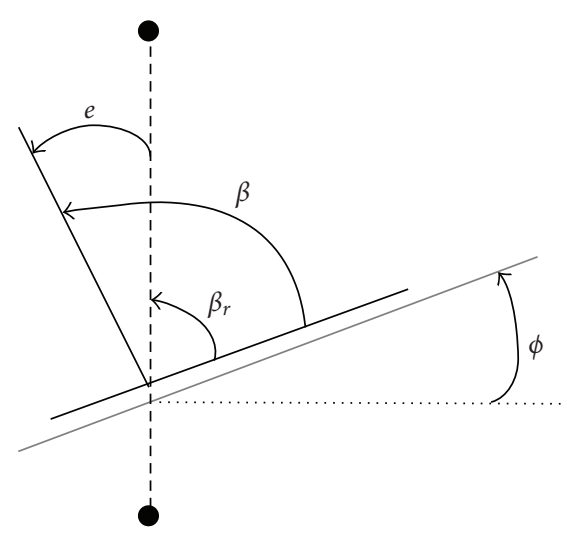

(a)

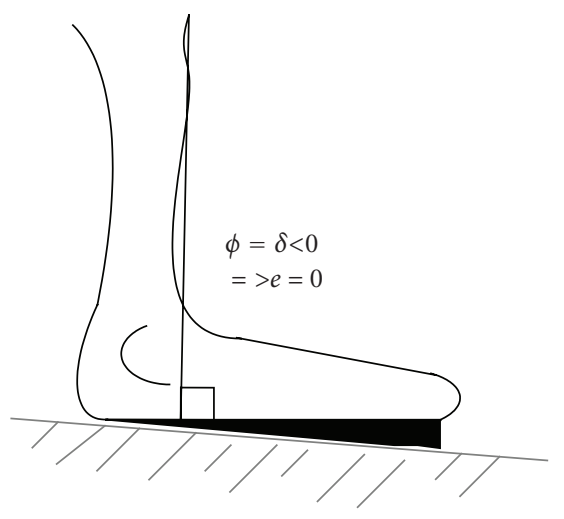

(b)

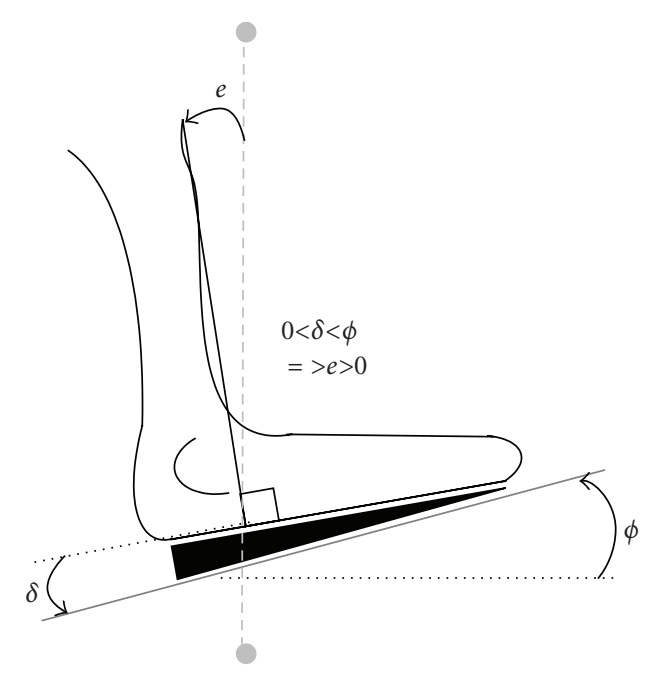

(c)

Figure 3: (a) Reference ankle angle $\beta_{r}$ and actual angle $\beta$ of an unloaded AFO give a control error $e$ in inclinations. Below: Ankle position of a biological foot during mid-stance while using compensating keels with resulting control error $e$. (b) The keel angle $\delta$ is chosen so that $e=0$ and (c) is the error shown as the angle between the actual orthosis and the vertical "desired" direction (dotted).

To quantify the estimation quality the root mean square error RMSE is used:

$$
\text { RMSE }=\sqrt{\frac{\sum_{k=0}^{M-1}\left(\phi_{k}-\hat{\phi}_{k}\right)^{2}}{M}} .
$$

3.4. Estimation of Control Error. The main motivation of this study was to find a way to estimate a control error suitable to be used for adjusting an ankle angle such that an orthosis or prosthesis can be designed to adapt to changes of ground slope inclinations. Previous studies have suggested a controller which tried to keep the lower leg motion adjusted to an imagined vertical line [20]. That suggests, as shown in Figure 3, that the AFO ankle angle $\beta$ is adjusted so that it during mid-stance, when it is unloaded, becomes equal to $\beta_{r}$. Thus, the system has an error defined as

$$
e(k)=\beta(k)-\beta_{r}(k)=\beta-\frac{\pi}{2}+\phi
$$

and a control law is then

$$
\beta(k+1)=\beta(k)-e(k) .
$$

Since the control error $e$ is not known, it needs to be estimated. Notice that when $\beta=\pi / 2$ it follows from (13) that $e=\phi$. Assume the strain changes in the same way by the ground inclination as by a corresponding change in ankle angle. If so, then the control error can be estimated by the same estimator as that used for inclination previously. Thus,

$$
\hat{e}(k)=x_{k}^{T} \theta
$$

which would be appropriate also for $\beta \neq \pi / 2$.

3.5. Experiment. Data sampling during gait was conducted by letting three healthy male subjects, whose heights are 182$186 \mathrm{~cm}$, walk continuously on a treadmill at different speeds and inclinations. Two test subjects A and B, wore their own walking shoes and one test subject $C$ used sandals with heel straps. 

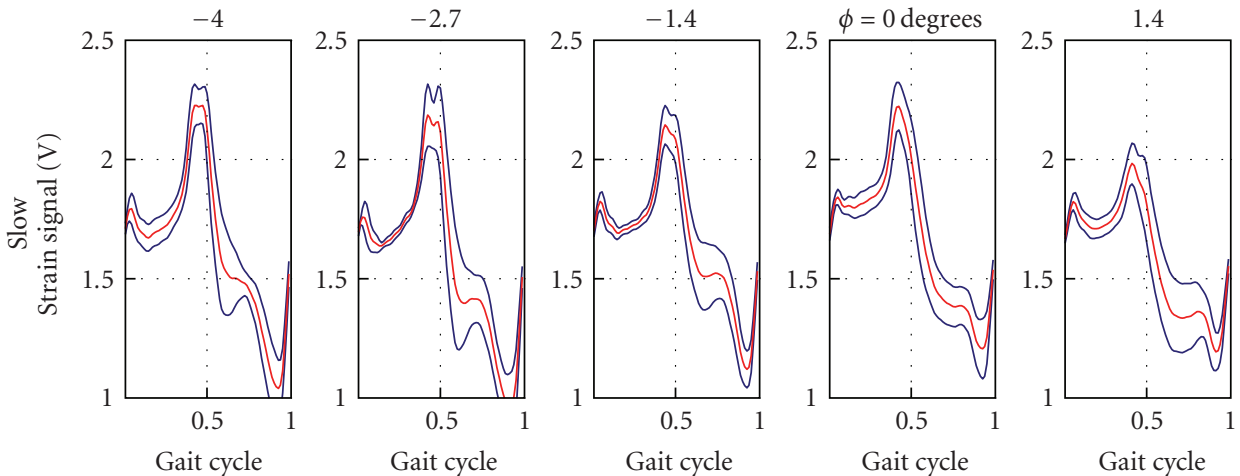

(a)
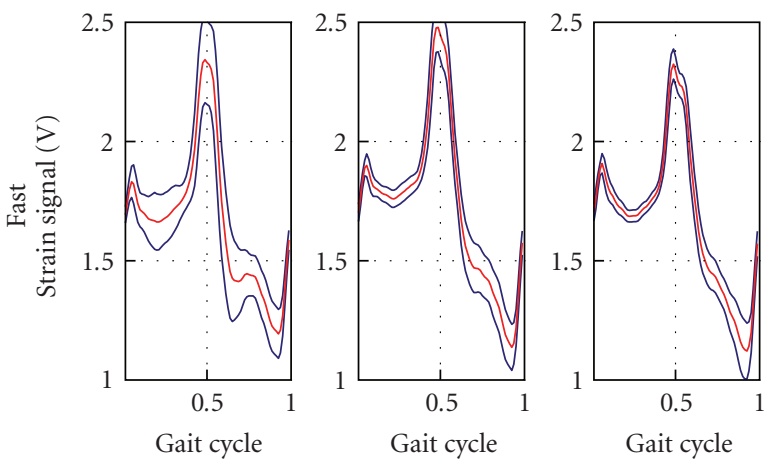
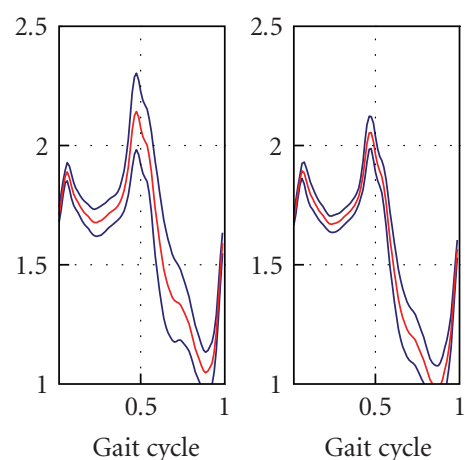

(b)

FIGURE 4: Mean strain signal (red) from test subject B, during a normalized gait cycle, from $t_{\text {start }}$ to $t_{\text {start }}$, at seven different ground inclinations $-4^{\circ}$ to $4^{\circ}$, and two speeds: slow speed $(0.5 \mathrm{~m} / \mathrm{s})$ and fast speed $(1.0 \mathrm{~m} / \mathrm{s})$. The standard deviations are plotted in blue.

First, gait experiments were made by letting subject $\mathrm{B}$ walk on the treadmill at inclination angles $0^{\circ}, \pm 1.4^{\circ}, \pm 2.7^{\circ}$, and $\pm 4^{\circ}$. For each inclination two speed conditions were investigated, $0.5 \mathrm{~m} / \mathrm{s}$ and $1.0 \mathrm{~m} / \mathrm{s}$, referred to hereafter as slow and fast. For each walking situation the gait cycles were extracted and the mean strain signal shape and its standard deviation were calculated. The purpose was to display the strain signal for various situations and attempt to explain intuitively characteristic differences.

Then, gait experiments were made on all subjects $\mathrm{A}$, $\mathrm{B}$ and $\mathrm{C}$, in order to investigate the performance of the inclination estimation technique. Ten different experiments were investigated for each one, consisting of the five inclinations $0^{\circ}, \pm 2.9^{\circ}$, and $\pm 4.7^{\circ}$ at the two speeds $0.5 \mathrm{~m} / \mathrm{s}$ (slow) and $1.0 \mathrm{~m} / \mathrm{s}$ (fast). Each walking condition was measured during $90 \mathrm{~s}$. Before measuring, each subject walked 10 steps on the treadmill getting familiar with the current speed and inclination. Three different inclination estimators were calculated for each subject. One used only slow speed data, the second used only fast speed data, and the third used both slow and fast speed data for the calculation of the estimator model parameter $\theta$. Since the third estimator is based on two different speeds, it becomes robust to speed variations. By comparing its performance to the estimators turned for one speed only, it is possible to find out what the robustness to speed variations costs in terms of degraded performance.
Data were split as $60 / 40 \%$ for estimation and validation, respectively.

Finally, experiments were made to validate the estimation of the control error. The AFO used in the experiments had a fixed ankle angle that could not be adjusted. Instead the ankle was adjusted by inserting a keel as shown in Figure 3. Then the error in (13) can be formulated as

$$
e=\phi-\delta
$$

where the $\delta$ is the keel angle. In this study the error was estimated from (15) and was compared with the calculated one using (16).

\section{Results}

4.1. Strain Signal Analysis. Uphill walking affected the strain signal curvature as expected. It was also observed that there was a considerable variation between steps, from heel strike to heel strike. If the ground angle increased, the plantarflexion peak was reduced in magnitude and almost disappeared completely for 4-degree uphill slow walking (Figure 4). This inclination also caused a large strain at dorsiflexion just before push-off. As previous studies have shown, an increase of dorsiflexion is a result of the compensation in the ankle foot system [4]. 


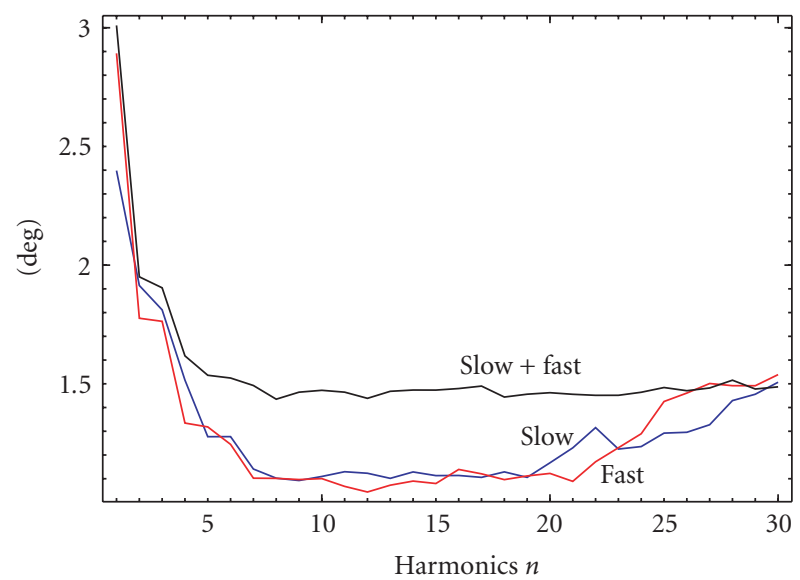

(a)

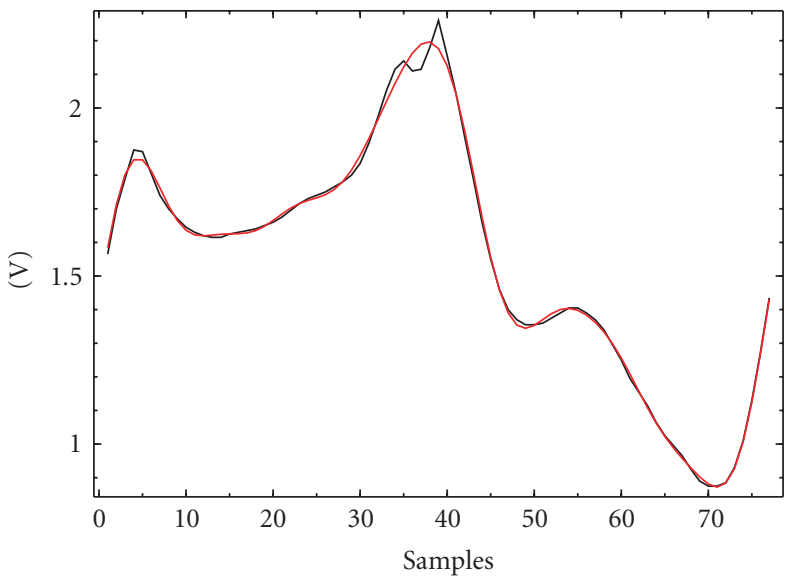

(b)

FIGURE 5: (a) RMSE for different Fourier series harmonics $n$, where the estimator is tuned for slow, fast, and the combined slow + fast walking speeds. No significant improvement is made for $n>8$. For $n=8$, the speed invariant estimator has RMSE $1.5^{\circ}$ compared to $1.2^{\circ}$ for the estimators specially tuned for slow or fast walking. (b) Measured strain signal s (black) and its Fourier series approximation $\hat{\mathbf{s}}$ (red), using $n=8$ harmonics.

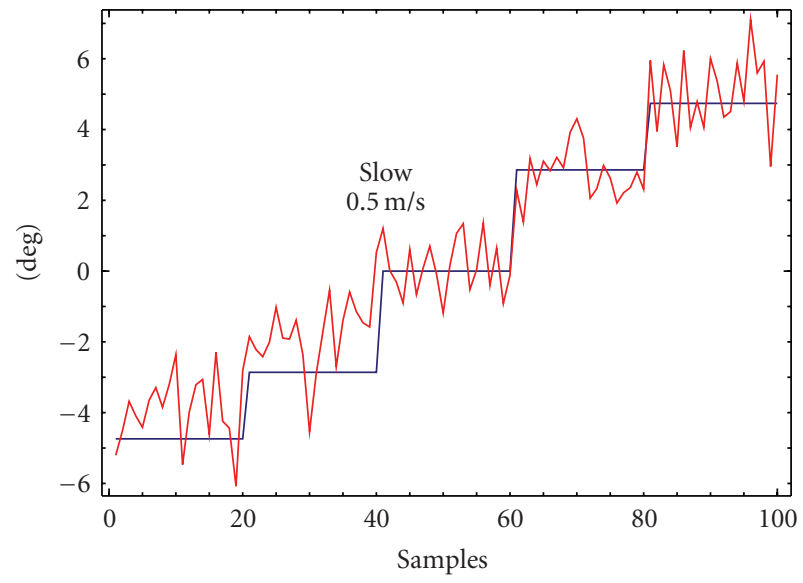

(a)

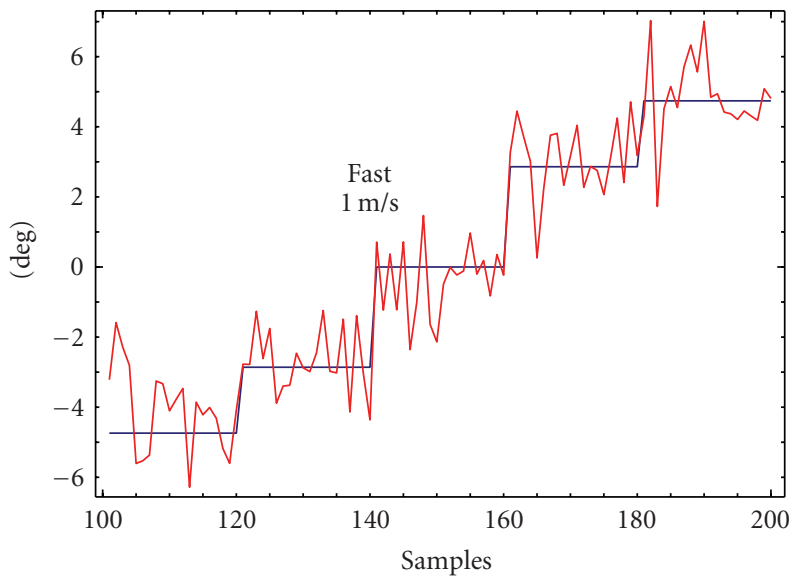

(b)

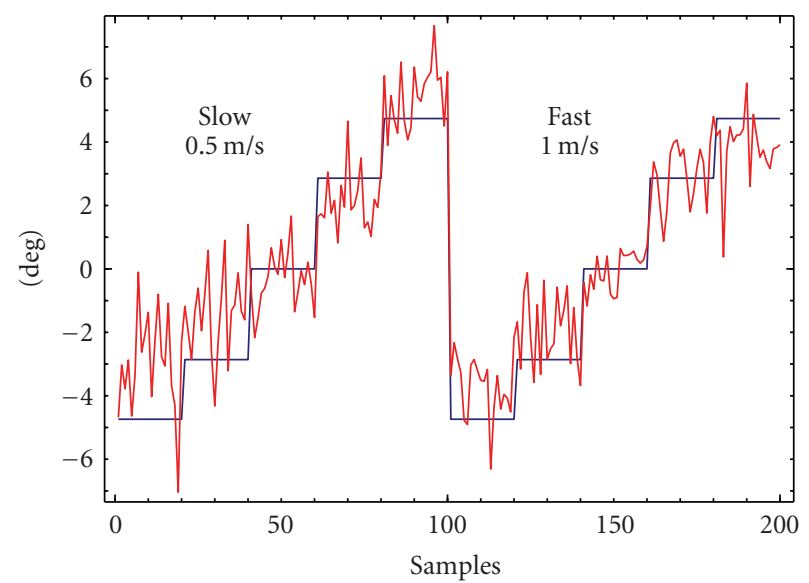

(c)

Figure 6: Ground angle estimation for subject B, tuned for (a) slow speed, (b) fast speed, and (c) slow + fast speeds. 


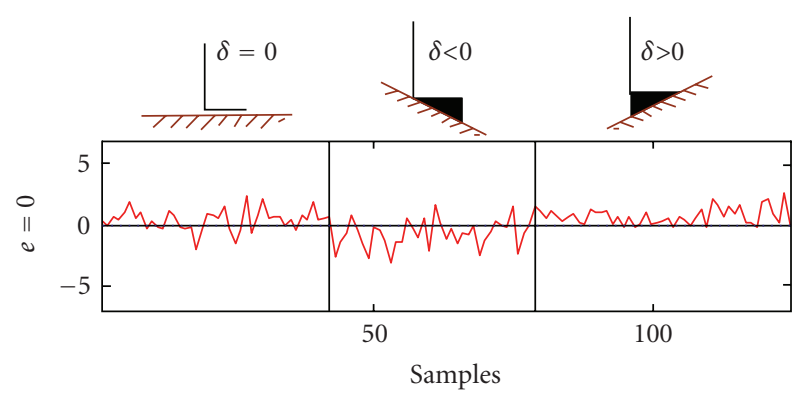

(a)

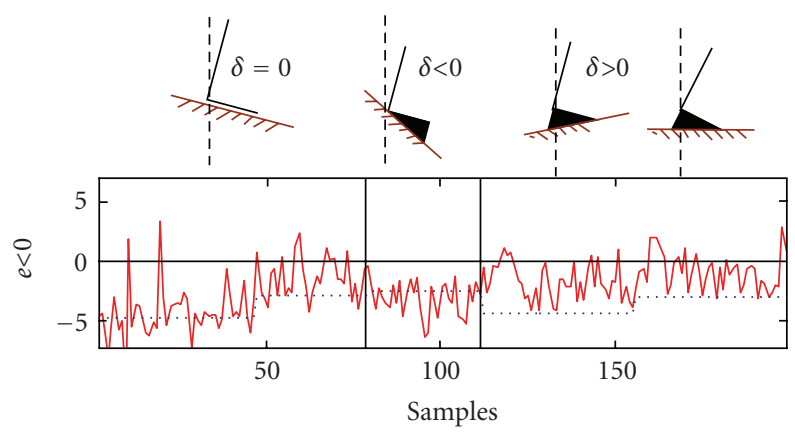

(b)

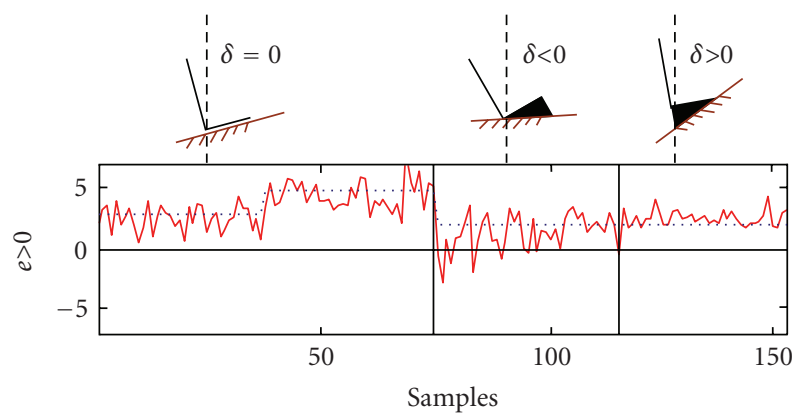

(c)

FIGURE 7: Control error of test subject B at different inclinations and control angles ( $\delta$ : keel angles), theoretical (dotted) calculated from (16) and estimated (solid) during fast walking using (15).

In downhill walking, the opposite foot has a lower position than the measured foot during weight transfer. Thus, the dorsiflexion should increase, which also can be observed in the strain signal. But there seems to be a difference between slow and fast walking in that the pushoff peak is increased for slow walking while the dorsiflexion strain has increased magnitude mostly at mid-stance for fast walking (Figure $4, \phi<0$ ). No significant difference in plantarflexion could be observed.

The differences between inclination and horizontal walking occur both at fast and slow walking. With increased speed, the step length increases which would cause a larger foot blade to ground ankle at heel strike. This is observed by the increase in plantarflexion for all inclinations (Figure 4, compare fast to slow). A faster gait also needs larger pushoff force, especially at uphill. This is seen as an increased dorsiflexion (Figure 4, compare fast to slow for $\phi>0$ ).
4.2. Estimation of Inclination. The ground angle estimation performance varied with the number of chosen Fourier harmonics $n$. RMSE for different harmonics $n$ of the gait cycle is shown in Figure 5(a), where it drops rapidly to a minimum around $n=8$. For higher harmonics, no significant improvement of the performance is seen Therefore the number of harmonics was chosen to be $n=8$, resulting in $\operatorname{dim}(x)=2 n+1=17$. The three different estimators, tuned for slow, fast, and the combination slow + fast walking speeds, can also be evaluated in Figure 5(a). At $n=8$, the RMSE is $1.2^{\circ}$ for the single speed estimators while it degrades to $1.5^{\circ}$ for the combined estimator based on both speeds. A rather modest cost to pay to get robustness against speed variations. The Fourier series approximated strain signal $\hat{s}$ for $n=8$ is compared to the measured strain signal $s$ in Figure 5(b). Clearly, most of the strain signal behavior is captured.

The three different inclination estimators, tuned for slow, fast, and the combined slow + fast walking situations are shown in Figures 6(a), 6(b), and 6(c), respectively. It is seen in Figure 6(c) that the combined estimator based on the two speeds mostly degrades at slow speed downhill.

The results for the other subjects $\mathrm{A}$ and $\mathrm{C}$ are similar and therefore not shown for the interest of brevity. The only difference in performance was noticed for subject $\mathrm{C}$ who walked using sandals, but surprisingly only with degraded performance for uphill walking $\left(\mathrm{RMSE}=2.3^{\circ}\right)$.

4.3. Estimation of Control Error. The control error estimator was validated at different inclinations and control angles (keel angles). The results show in Figure 7 that the error has similar precision as when estimating ground inclination. Walking with a keel which has the same angle as the ground clearly centralizes the estimations around zero. The estimator also finds the right sign of the error for other walking situations, which is essential for control law (14).

\section{Conclusions}

An embedded measurement system for foot orthosis during gait is proposed. Strain gauge sensors were mounted on a foot orthosis in order to give information about strain in the sagittal plane. It was shown that the extracted strain signal for each gait cycle can be efficiently coded into a vector representation based on Fourier series. This vector has a fixed and lower dimension than the original strain signal. It can be used in linear regression techniques for estimation of ground slope inclination. The proposed estimation scheme uses the entire gait cycle as opposed to kinematically based estimators that need a stationary phase in order to extract inclination information from accelerometers. A drawback could be that the strain signal-based estimator needs to be tuned for each person's walking style. A particular interesting feature, though, is that a control error defined as the unloaded orthosis deviation from the vertical line can be estimated. The orthosis used here was not hinged and the ankle angle could not be changed. A keel was used to artificially change the ankle angle to a "controlled" position. It was then verified 
that the inclination estimator produced estimates of the control error. This error is thereby controllable. A future development of a hinged actuated orthosis would be able to take a advantage of the proposed technique for online ankle angle adaptation. Also, as opposed to kinematically based estimators this approach does not need to be recalibrated when changing shoes with other heel heights. Further tests on disabled are now needed for more detail evaluation.

\section{References}

[1] J. A. Blaya and H. Herr, "Adaptive control of a variableimpedance ankle-foot orthosis to assist drop-foot gait," IEEE Transactions on Neural Systems and Rehabilitation Engineering, vol. 12 , no. 1, pp. 24-31, 2004.

[2] J. C. Moreno, F. J. Brunetti, J. L. Pons, J. M. Baydal, and R. Barberà, "Rationale for multiple compensation of muscle weakness walking with a wearable robotic orthosis," in Proceedings of IEEE International Conference on Robotics and Automation, pp. 1914-1919, Barcelona, Spain, April 2005.

[3] A. B. Zoss, H. Kazerooni, and A. Chu, "Biomechanical design of the berkeley lower extremity exoskeleton (bleex)," IEEE/ASME Transactions on Mechatronics, vol. 11, no. 2, pp. 128-138, 2006.

[4] A. H. Hansen, D. S. Childress, and S. C. Miff, "Rollover characteristics of human walking on inclined surfaces," Human Movement Science, vol. 23, no. 6, pp. 807-821, 2004.

[5] A. Leroux, J. Fung, and H. Barbeau, "Postural adaptation to walking on inclined surfaces: I. Normal strategies," Gait \& Posture, vol. 15, no. 1, pp. 64-74, 2002.

[6] M. S. Redfern and J. DiPasquale, "Biomechanics of descending ramps," Gait \& Posture, vol. 6, no. 2, pp. 119-125, 1997.

[7] M. Kuster, S. Sakurai, and G. A. Wood, "Kinematic and kinetic comparison of downhill and level walking," Clinical Biomechanics, vol. 10, no. 2, pp. 79-84, 1995.

[8] A. S. McIntosh, K. T. Beatty, L. N. Dwan, and D. R. Vickers, "Gait dynamics on an inclined walkway," Journal of Biomechanics, vol. 39, no. 13, pp. 2491-2502, 2006.

[9] B. T. Smith, D. J. Coiro, R. Finson, R. R. Betz, and J. McCarthy, "Evaluation of force-sensing resistors for gait event detection to trigger electrical stimulation to improve walking in the child with cerebral palsy," IEEE Transactions on Neural Systems and Rehabilitation Engineering, vol. 10, no. 1, pp. 22-29, 2002.

[10] M. M. Skelly and H. J. Chizeck, "Real-time gait event detection for paraplegic FES walking," IEEE Transactions on Neural Systems and Rehabilitation Engineering, vol. 9, no. 1, pp. 5968, 2001.

[11] K. Aminian, B. Najafi, C. Büla, P.-F. Leyvraz, and P. Robert, "Spatio-temporal parameters of gait measured by an ambulatory system using miniature gyroscopes," Journal of Biomechanics, vol. 35, no. 5, pp. 689-699, 2002.

[12] I. P. I. Pappas, M. R. Popovic, T. Keller, V. Dietz, and M. Morari, "A reliable gait phase detection system," IEEE Transactions on Neural Systems and Rehabilitation Engineering, vol. 9, no. 2, pp. 113-125, 2001.

[13] R. Williamson and B. J. Andrews, "Gait event detection for FES using accelerometers and supervised machine learning," IEEE Transactions on Rehabilitation Engineering, vol. 8, no. 3, pp. 312-319, 2000.

[14] A. T. M. Willemsen, F. Bloemhof, and H. B. K. Boom, "Automatic stance-swing phase detection from accelerometer data for peroneal nerve stimulation," IEEE Transactions on Biomedical Engineering, vol. 37, no. 12, pp. 1201-1208, 1990.
[15] A. M. Sabatini, C. Martelloni, S. Scapellato, and F. Cavallo, "Assessment of walking features from foot inertial sensing," IEEE Transactions on Biomedical Engineering, vol. 52, no. 3, pp. 486-494, 2005.

[16] S. K. Ng and H. J. Chizeck, "Fuzzy model identification for classification of gait events in paraplegics," IEEE Transactions on Fuzzy Systems, vol. 5, no. 4, pp. 536-544, 1997.

[17] K. Aminian, P. Robert, E. Jéquier, and Y. Schutz, "Estimation of speed and incline of walking using neural network," IEEE Transactions on Instrumentation and Measurement, vol. 44, no. 3, pp. 743-746, 1995.

[18] N. Wang, E. Ambikairajah, N. H. Lowell, and B. G. Celler, "Accelerometry based classfication of walking patterns using time-frequency analysis," in Proceedings of the 29th Annual International Conference of the IEEE Engineering in Medicine and Biology Society, Lyon, France, August 2007.

[19] W. Svensson and U. Holmberg, "Ground angle estimator from an ankle foot orthosis based on strain sensing and Fourier series," in Proceedings of IEEE International Conference on Mechatronics and Automation (ICMA '08), pp. 203-206, Takamatsu, Japan, August 2008.

[20] W. Svensson and U. Holmberg, "An autonomous control system for prosthetic foot ankle," in Proceedings of the 4th IFAC Symposium on Mechatronic Systems, pp. 856-861, Heidelberg, Germany, September 2006. 

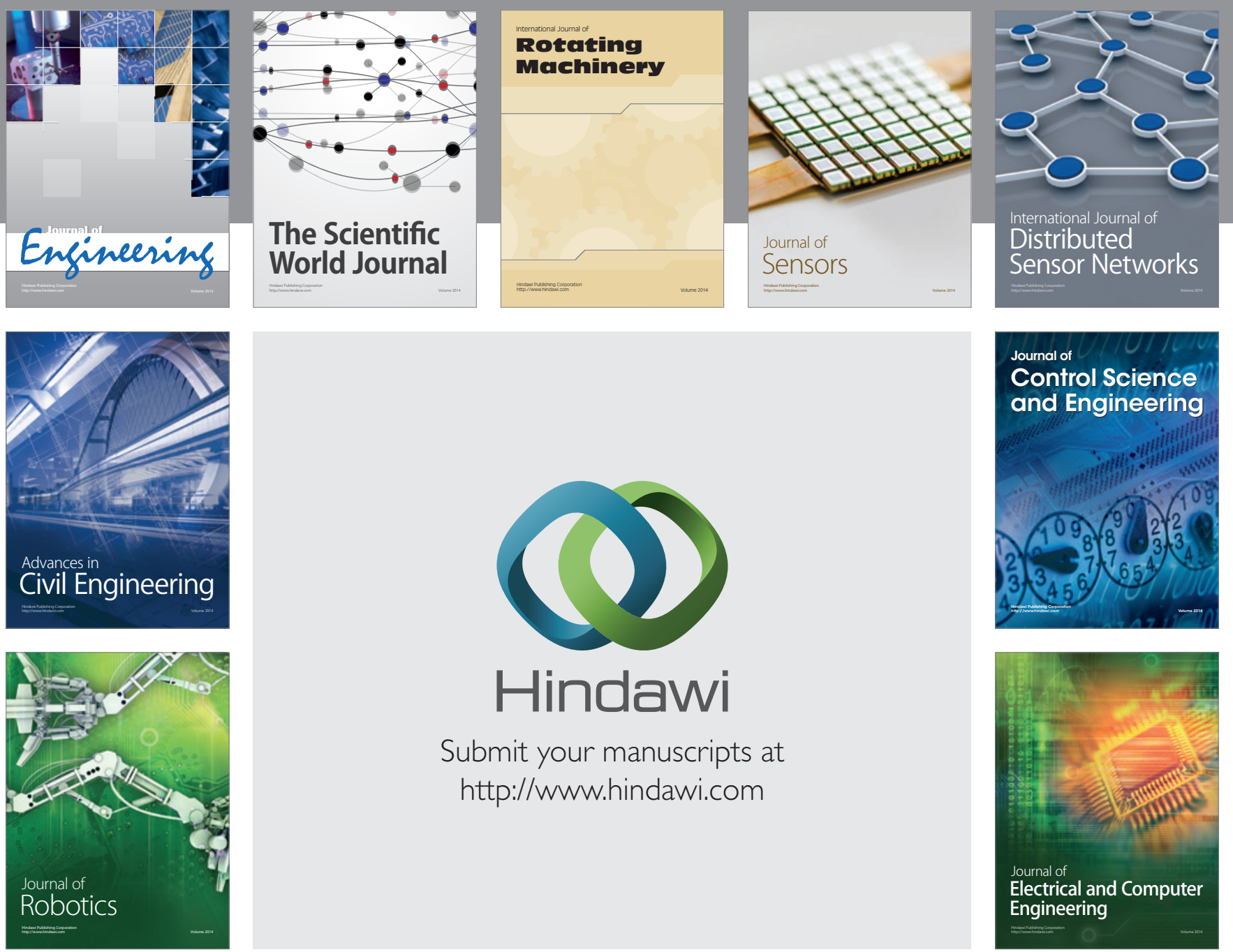

Submit your manuscripts at

http://www.hindawi.com
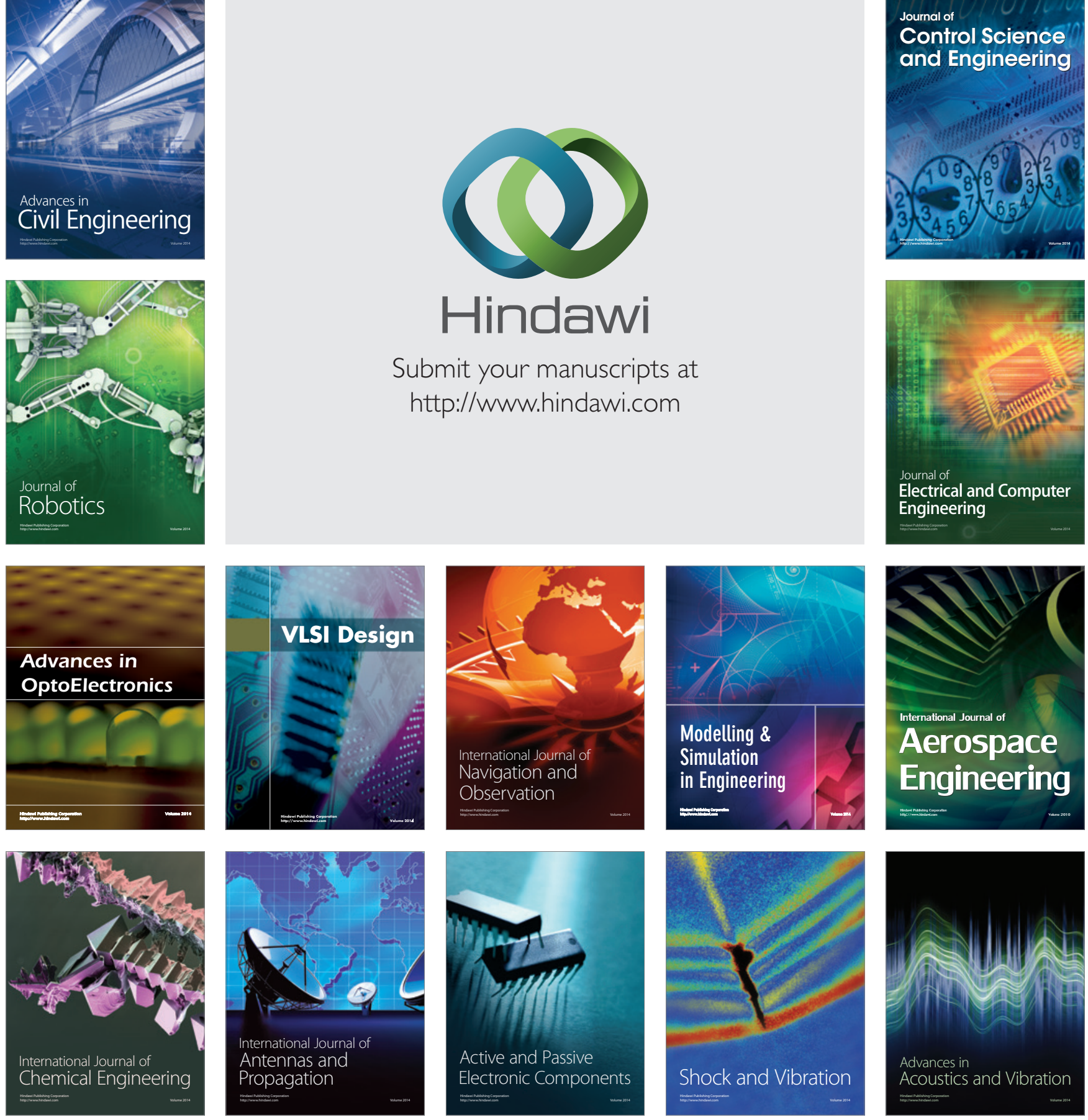